\title{
Benefícios de um programa de educação postural para alunos de uma escola municipal de Garibaldi, RS
}

\author{
Benefits of a posture education program for schoolchildren in the city of Garibaldi, \\ $R S$
}

Estudo desenvolvido no Curso de Fisioterapia da Unisinos Universidade do Vale do Rio dos Sinos, São Leopoldo, RS, Brasil

\section{Fisioterapeuta}

2 Fisioterapeuta; Profa. MS. do Curso de Fisioterapia da Unisinos

\section{ENDERECO PARA CORRESPSONDÊNCIA:}

Ana Paula B. Karolczak R: Vicente da Fontoura 1831 ap.103 Rio Branco 90640003 Porto Alegre RS e-mail: anapbk@gmail.com

\section{APRESENTAÇ̃̃O mar. 2010}

\section{ACEITO PARA PUBLICAÇÃO} set. 2010

\author{
Juliana Benini', Ana Paula Barcellos Karolczak ${ }^{2}$
}

Resumo: A fisioterapia tem importante papel no meio escolar, pois estudantes desenvolvem maus hábitos e alterações posturais que, a longo prazo, podem gerar restrição funcional. O objetivo deste estudo foi analisar os efeitos de um programa de educação postural em estudantes do ensino fundamental da cidade de Garibaldi, RS. Participaram 48 alunos com idade entre 8 e 10 anos, que responderam um questionário sobre hábitos posturais e foram submetidos à avaliação de peso, modelo e modo de transporte da mochila antes e após uma sessão educativa; quatro semanas mais tarde foi feita uma reavaliação. Os pais participaram do estudo respondendo um questionário sobre a postura dos filhos. Quanto aos hábitos escolares, constatouse mudança positiva na adoção de postura adequada dos pés na posição sentada $(p=0,001)$; e, nas atividades de vida diária, mudanças na posição ao ver televisão $(p<0,0001)$, dormir $(p=0,019)$, pegar objetos no chão $(p<0,0001)$ e costume de ler e/ou escrever na cama $(p=0,002)$. Em relação ao peso da mochila obteve-se redução significativa após a intervenção $(p=0,002)$, enquanto no modelo e modo de transporte não houve diferença. Segundo os pais, os filhos têm bons hábitos de postura e costumam utilizá-los no dia-a-dia. Concluiu-se que a realização de uma sessão de educação postural para alunos promoveu o conhecimento de hábitos posturais saudáveis e modificação de algumas posturas, mas não se pode afirmar que provocou mudanças nos hábitos posturais.

Descritores: Criança; Estudantes; Fisioterapia (especialidade); Postura; Promoção da saúde

Abstract: Physical therapy plays an important role in school, as students develop bad habits and postural dysfunctions which may generate long-term functional restrictions. The aim of this study was to analyse the effects of an educational program on students' posture in Garibaldi, RS. Forty-eight 8-to-10 year-old schoolchildren filled up a questionnaire about postural habits and had their knapsacks assessed as to weight, type and carrying mode, before and after an educational session; a further assessment was made four weeks later. Parents participated by answering a questionnaire on children's posture. Results showed positive changes in feet posture in the sitting position $(p=0.001)$; in daily living activities, better postures or habits were found while watching television $(p<0.0001)$, sleeping $(p=0.019)$, getting objects from the ground $(p<0.0001)$, and reading and/or writing in bed $(p=0.002)$. As to knapsack weight, a significant decrease $(p=0,002)$ was noticed after the session, while neither the model or mode of transport changed. Parents answered that their children have good postural habits and keep them on a regular basis. It may thus be said that one session of postural educational program for schoolchildren resulted in better knowledge on healthy postural behaviour and modified some postures, but one can't say it brought about changes in postural habits.

Key words: Child; Health promotion; Physical therapy (speciality); Posture; Students 


\section{INTRODUÇÃO}

A carta de Ottawa define promoção da saúde como "o processo de capacitação da comunidade para atuar na melhoria de sua qualidade de vida e saúde" 1 . Programas educativos agem sobre os riscos comportamentais, que podem ser modificados, pois em parte estão sob o controle das pessoas ${ }^{2}$. Nesse sentido, tem-se buscado estudar a saúde escolar no que concerne às alterações posturais e dores na coluna de crianças e adolescentes, principalmente entre os 7 e 14 anos de idade, em decorrência do grande número de adultos acometidos 3,4 .

A postura é uma posição ou atitude do corpo para uma atividade específica, ou para sustentá-lo ${ }^{5}$. A boa postura refere-se àquela que a pessoa mantém com esforço muscular mínimo, estabelecendo uma maneira individual de sustentação do corpo, orientada em função da linha de gravidade. Em contrapartida, a má postura aumenta o estresse sobre elementos corporais, e o distribui para estruturas menos capazes de suportá-lo, provocando mudança no centro de gravidade e, em conseqüência, alterações posturais e dor6.

Os problemas posturais comumente aparecem com o crescimento, em decorrência dos comportamentos de risco adotados para a coluna vertebral, como a utilização indevida das mochilas e a má postura ao sentar7. A escoliose, por exemplo, é uma condição que atinge mais de $20 \%$ da população infantil, instalada por uma causa inicial associada ao estirão de crescimento; contudo, em 75\% dos casos, poderia ser evitada pela educação postural iniciada precocemente ${ }^{8}$.

Os maus hábitos posturais constituem problema de saúde coletiva, atingindo estudantes de ambos os sexos em diferentes idades, acabando por instigar fisioterapeutas e demais profissionais da saúde a propor programas educativos para a busca de hábitos posturais saudáveis, bem como prevenir futuras complicações 6 .

Diversos programas de educação postural são propostos na literatura3,9-15. Destaca-se o método intitulado "Escola das costas" (Back school), desenvolvido em 1969 na Suécia pela fisioterapeuta Mariane Zachrisson-Forsell, que propõe um programa de quatro aulas de cerca de 45 minutos, duas vezes por semana. As aulas consistem em divulgar medidas para prevenir a incidência de dor na coluna, incluindo conhecimentos de anatomia, biomecânica e fisiopatologia das dores dessa região. Além disso, orientações ergonômicas e posturais das atividades ocupacionais e de vida diária, exercícios de alongamento, força e relaxamento musculares são passados aos participantes 15. No Brasil, a Back school tem recebido diferentes denominações: uma delas foi "Escola de postura", introduzida em 1972 no Hospital do Servidor Público Estadual de São Paulo por Knoplich, a partir da observação de que, no Departamento de Ortopedia, mais de $80 \%$ dos pacientes apresentavam queixas de dor crônica na coluna, o que aumentava a demanda tanto do atendimento médico como da fisioterapia 16 .

Cardon, De Clercq e De Bourdeaudhuij publicaram vários estudos sobre programas de educação postural que aplicaram a escolares17-19. Em um desses, observaram modificações significativas no conhecimento sobre cuidados com a coluna vertebral, confirmados em seguimento de um ano ${ }^{18}$. Achados semelhantes foram descritos em outros estudos, e os resultados revelam modificações significativas no conhecimento da saúde da coluna e das posturas em geral, no modo de transporte das mochilas escolares, e na carga transportada.

Embora a literatura internacional seja extensa em relação à educação postural de escolares, evidencia-se a escassez de estudos envolvendo a população brasileira, cujos hábitos e condições de saúde podem não ser semelhantes aos de outros países ${ }^{3}$. Assim, o presente estudo teve como objetivo analisar os benefícios de um programa de educação postural em estudantes de uma escola de ensino fundamental.

\section{METODOLOGIA}

Este estudo é caracterizado como experimental longitudinal, do tipo antes e depois. Foi aprovado pelo Comitê de Ética em Pesquisa da Universidade do Vale do Rio dos Sinos e segue os preceitos éticos recomendados pelo Conselho Nacional de Saúde.
Participaram da pesquisa estudantes de ambos os sexos, com idade entre $8 \mathrm{e}$ 10 anos, de uma escola de ensino fundamental da rede municipal da cidade de Garibaldi, RS. Os critérios de inclusão adotados foram: estudantes alfabetizados com habilidade para leitura e escrita, que compareceram às duas avaliações, responderam os questionários antes e depois da intervenção, e de cujos pais ou responsáveis foi obtido o consentimento assinado quanto à participação no estudo. Foram excluídos os estudantes que apresentaram doença musculoesquelética, neuromuscular ou cirurgias ortopédicas.

\section{Procedimentos}

Os alunos responderam um questionário 6,9 aplicado em sala de aula, com questões fechadas sobre hábitos posturais na escola (postura sentada, modelo e modo de transporte da mochila) e nas atividades de vida diária (AVD - postura ao assistir televisão, dormir, pegar um objeto do chão, costume de ler ou escrever na cama). Os pais também receberam um questionário, para se verificar sua percepção em relação aos hábitos posturais dos filhos.

As crianças foram inicialmente medidas quanto à massa corporal e estatura (balança Cauduro, modelo B160, Cachoeira do Sul, RS); as mochilas foram avaliadas quanto ao peso (balança Yara, Vulcão SA, SP), quanto ao modelo (uma alça, duas alças, carrinho) e modo de utilização (ombro unilateral, bilateral, na mão, carrinho, transpassada). Em seguida, participaram de uma sessão educativa. Foram reavaliadas quatro semanas depois, no mesmo dia da semana da avaliação inicial, de forma a garantir que os alunos estivessem carregando os mesmos materiais.

Durante a sessão educativa, os professores foram convidados a participar, para aprenderem e divulgarem os bons hábitos posturais e, também, para capacitá-los a dar prosseguimento. Primeiramente foram abordadas a anatomia e as lesões da coluna vertebral e, em seguida, informações sobre o excesso de carga transportada, o modelo de mochila de duas alças e o modo de transporte da mochila, nas costas com apoio bilateral, considerado o mais adequado na lite- 
ratura 3,4 . Foram passadas as seguintes orientações sobre bons hábitos: sentar na cadeira com as costas apoiadas no encosto e os pés apoiados no chão ou em uma caixinha; carregar a mochila nas costas e com apoio bilateral; dormir na postura de lado; pegar um objeto do chão sempre flexionando os joelhos e não a coluna; e não adotar o hábito de ler e/ou escrever na cama, fazendo isso em uma mesa, sentado em uma cadeira. Foi utilizada uma linguagem apropriada, a fim de facilitar a compreensão por parte dos estudantes, sendo apresentados cartazes de apoio ilustrados. O componente prático teve como objetivo o treino dos hábitos posturais corretos, por meio de uma dinâmica em sala de aula simulando as posturas corretas ao realizar as tarefas, além da construção, em conjunto, de um cartaz com figuras das posturas adequadas e inadequadas, que posteriormente foi fixado em local escolhido pelos estudantes. Ao final, cada aluno recebeu um folheto com orientações sobre hábitos posturais 10 , que também foi entregue aos pais ou responsáveis.

\section{Análise estatística}

As variáveis foram analisadas descritivamente. por meio da média e desvio padrão, bem como de freqüências absolutas e relativas. Para verificar a associação entre as variáveis categóricas com mais de duas categorias, antes e após a intervenção, utilizou-se o teste de associação do qui-quadrado, de Pearson. As diferenças entre variáveis dicotômicas antes e depois da intervenção foram calculadas pelo teste de McNemar. A comparação das variáveis numéricas relacionadas foi feita pelo teste de Wilcoxon para dados pareados. Foi utilizado o programa SPSS (Statistical Package For Social Sciences v.17.0), sendo adotado o nível de significância estatística $p \leq 0,05$.

\section{RESULTADOS}

A amostra foi constituída por 48 estudantes, sendo 50\% meninas e 50\% meninos, matriculados na $2 \underline{a}(23 \%), 3 \underline{a}$ $(37 \%)$ e 4 a séries $(40 \%)$ do ensino fundamental. Na faixa de 8 anos estavam $45,8 \%$ dos estudantes; $37,5 \%$ tinham 9 anos e 16,7\%, 10 anos. A massa corporal média foi de $33,2 \pm 8,11 \mathrm{~kg}$ e a estatura, de $132,2 \pm 6,72 \mathrm{~cm}$.
Na Tabela 1 são apresentados os resultados referentes à postura adotada nas atividades escolares e AVD, antes e depois da sessão educativa. Pôde-se constatar que a posição dos pés quando sentado passou de "pendurados" para "alcançam o chão com as pontas" $(p=0,001)$. A postura adotada ao assistir televisão passou de "deitada na cama" para "sentada com as costas apoiadas" $(p<0,001)$. Ao dormir, em média já adotavam a postura "de lado", mantendo essa posição após a intervenção $(p=0,019)$.

Os resultados quanto às posturas habituais nas AVD são apresentados na Tabela 2. Nota-se que o costume de estudarem sentados ou deitados diminuiu significativamente $(p=0,002)$; da mesma forma, o ato de pegar um objeto do chão flexionando a coluna passou a ser realizado com flexão dos joelhos ( $\mathrm{p}<0,0001$ ).

O peso das mochilas apresentou redução significativa, de $2,85 \pm 0,98 \mathrm{~kg}$ para $2,38 \pm 0,76 \mathrm{~kg}(p=0,002)$; entretanto, os resultados quanto ao modelo e modo de carregar a mochila não se apresentaram significativamente diferentes (Tabela 3).

Tabela 1 Distribuição (n, \%) dos hábitos posturais antes e depois da sessão educativa e valor de $\mathrm{p}$ da comparação entre os dois momentos $(n=48)$

\begin{tabular}{lccccc}
\hline \multirow{2}{*}{ Postura } & \multicolumn{2}{c}{ Antes } & \multicolumn{2}{c}{ Depois } & \multirow{2}{*}{$p$} \\
& $\mathrm{n}$ & $\%$ & $\mathrm{n}$ & $\%$ & $p$ \\
\hline Posição da coluna na postura sentada & & & & & 0,273 \\
Reta, encostada na cadeira & 38 & 79,2 & 35 & 72,9 & \\
Curvada, encostada na cadeira & 8 & 16,7 & 7 & 14,6 & \\
Curvada longe da cadeira & 2 & 4,2 & 4 & 8,3 & \\
Não sabe & 0 & 0 & 2 & 4,2 & \\
Posição dos pés na postura sentada & & & & & 0,001 \\
Alcançam o chão, pés apoiados & 10 & 20,8 & 12 & 25,0 & \\
Pés pendurados & 18 & 37,6 & 10 & 20,8 & \\
Alcançam o chão, sentado na beira da cadeira & 10 & 20,8 & 4 & 8,3 & \\
Só as pontas dos pés alcançam o chão & 10 & 20,8 & 22 & 45,8 & \\
Posição ao ver televisão & & & & & 0,0001 \\
Sentado, costas apoiadas & 13 & 27,1 & 18 & 37,5 & \\
Sentado, costas escorregando pelo sofá & 8 & 16,7 & 8 & 16,7 & \\
Deitado na cama & 21 & 43,8 & 14 & 29,2 & \\
Outra & 6 & 12,5 & 8 & 16,7 & \\
Posição em que mais gosta de dormir & & & & & 0,019 \\
Barriga para baixo & 8 & 16,7 & 8 & 16,7 & \\
Barriga para cima & 6 & 12,5 & 0 & 0 & \\
De lado & 34 & 70,8 & 40 & 83,3 & \\
\hline
\end{tabular}

Tabela 2 Distribuição (n, \%) de posturas habituais em AVD antes e depois da sessão educativa e valor de p da comparação entre os dois momentos $(\mathrm{n}=48)$

\begin{tabular}{lccccc}
\hline \multirow{2}{*}{ Atividade } & \multicolumn{2}{c}{ Antes } & \multicolumn{2}{c}{ Depois } & \multicolumn{1}{c}{$p$} \\
\hline Costume de ler/escrever na cama & $\mathrm{n}$ & $\%$ & $\mathrm{n}$ & $\%$ & \\
$\quad$ Não & 20 & 41,7 & 33 & 68,8 & \\
Sim & 28 & 58,3 & 15 & 31,3 & \\
Pegar objetos do chão & & & & & 0,000 \\
$\quad$ Dobrando as costas & 31 & 64,6 & 10 & 20,8 & \\
Dobrando os joelhos & 17 & 35,4 & 38 & 79,2 & \\
\hline
\end{tabular}

AVD = atividades da vida diária 
Tabela 3 Distribuição (n, \%) do modelo e modo de carregar a mochila antes e depois da sessão educativa e valor de $p$ da comparação entre os dois momentos $(n=48)$

\begin{tabular}{lccccc}
\hline \multirow{2}{*}{ Mochila } & \multicolumn{2}{c}{ Antes } & \multicolumn{2}{c}{ Depois } & \\
& $\mathrm{n}$ & $\%$ & $\mathrm{n}$ & $\%$ & $p$ \\
\hline Modelo da mochila & & & & & \\
$\quad$ Uma alça & 8 & 16,7 & 9 & 18,8 & $\mathrm{~N} \mathcal{F}^{*}$ \\
$\quad$ Duas alças & 36 & 75,0 & 35 & 72,9 & $\mathrm{NS}$ \\
$\quad$ Carrinho & 4 & 8,3 & 4 & 8,3 & $\mathrm{NS}$ \\
Modo de transporte & & & & & \\
$\quad$ Nas costas, bilateral & 30 & 62,5 & 35 & 72,9 & $\mathrm{NS}$ \\
$\quad$ Nas costas, unilateral & 1 & 2,1 & 1 & 2,1 & $\mathrm{NS}$ \\
$\quad$ Segurando na mão & 6 & 12,5 & 0 & 0,0 & $\mathrm{NS}$ \\
$\quad$ Outro & 11 & 22,9 & 12 & 25,0 & $\mathrm{NS}$ \\
\hline
\end{tabular}

No que se refere à percepção dos pais ou responsáveis quanto à postura das crianças, 60,4\% responderam que os filhos costumam sentar-se com a coluna reta e encostada na cadeira; 39,6\%, que se sentam com os pés retos e apoiados no chão; e 68,8\%, que transportam a mochila nas costas bilateralmente. A respeito da postura dos filhos ao assistir televisão, $39,6 \%$ dos pais afirmaram que os filhos ficam sentados com as costas escorregando pelo encosto do sofá; $52,1 \%$, que costumam pegar os objetos flexionando os joelhos; e $81,3 \%$ referiram que os filhos adotam a postura de dormir de lado. Quanto aos hábitos posturais dos filhos em geral, 58,3\% responderam que apresentam bons hábitos posturais; e, por fim, 50\% dos pais disseram ter conhecimento das posturas corretas.

\section{DISCUSSÃO}

Durante o período letivo, estudantes cumprem uma rotina de transporte da mochila e prolongados períodos sentados que, aliados a proporções inadequadas do mobiliário em geral oferecido pelas escolas 3 , são considerados fatores responsáveis pela aquisição, manutenção ou agravamento de hábitos posturais inapropriados, expondo a coluna vertebral a desequilíbrios musculares e alterações de estruturas anatômicas em fase de crescimento $3,10,21,22$. Por outro lado, o período escolar é favorável para a percepção precoce de alterações posturais, bem como para sua prevenção 23 .

O presente estudo teve o propósito de analisar a influência de uma sessão de educação postural sobre os hábitos posturais de escolares. Constatou-se que os alunos têm conhecimento da maneira correta de sentar-se para assistir às aulas, embora não a ponham em prática. Na postura utilizada para assistir televisão, obteve-se aumento na adoção do apoio da coluna no encosto do sofá; também o costume de ler na cama foi modificado de forma positiva. Zapater et al.10 aplicaram um programa de educação postural em escolares de Bauru, SP e, apesar de realizar apenas uma sessão educativa, contaram com reforço por parte dos professores. Tal como no presente estudo, verificaram boa aceitação por parte dos escolares, uma vez que proporcionou aumento do conhecimento da postura sentada adequada, mas não podem afirmar se houve mudança de hábitos, e sugerem acompanhamento por maior tempo.

A posição sentada é considerada a mais prejudicial para a coluna, provocando aumento da pressão no interior dos discos intervertebrais; porém, sentar-se apoiando o tronco diminui a pressão intradiscal e parte do peso corporal é transferida para o encosto 24 ; assim, o hábito de sentar na cama, no sofá, sem um bom apoio para a coluna poderá promover aumento das curvaturas da coluna vertebral. A não-exposição da coluna a posturas inadequadas por longos períodos pode prevenir alterações posturais e sintomatologia dolorosa associada. Em seu estudo também no Rio Grande do Sul, Detsch6 encontrou que $90 \%$ dos estudantes adotam posição incorreta durante as aulas, e 93\% ao assistir televisão ${ }^{6}$.

Entretanto, parece não ser suficiente a orientação quanto à postura sentada correta, uma vez que se sabe que o mobiliá- rio inadequado constitui importante fator predisponente para a adoção da postura sentada relaxada, com apoio sacral ${ }^{9}$. Também Limon et al.25 identificaram como fatores de risco para dor lombar em estudantes as cadeiras de sala de aula inapropriadas, a posição sentada em ângulo desconfortável para visualização do professor, o carregar mais de $15 \%$ do peso corporal nas mochilas, e educação física não oferecida durante os intervalos. Pôde-se observar no presente estudo que o mobiliário não é ajustável à estatura dos estudantes, tornando-se incômodo para ficarem sentados na mesma posição durante horas. É natural, portanto, que os alunos busquem posturas mais cômodas - e por vezes inadequadas para a saúde postural26-, mesmo detendo o conhecimento da postura sentada correta.

Por outro lado Skoffer27, ao avaliar dor lombar em estudantes de 15 a 16 anos, não encontrou correlação com o mobiliário, mas sim com o fato de carregarem a mochila de modo assimétrico. Esse resultado talvez tenha relação com a idade superior dos estudados, comparada à do presente estudo, e o mobiliário ser menos apropriado às medidas antropométricas de alunos menores.

$\mathrm{O}$ ato de pegar um objeto do chão de maneira correta foi bem fixado pelos alunos, assim como a postura dormir de lado; acredita-se que isso se deva ao fato de terem sido realizadas demonstrações práticas dessas posturas. Méndez e Gómes-Conesa ${ }^{12}$ ressaltaram a importância de práticas em detrimento da mera "passagem" de informações; observaram mudanças dos hábitos posturais, inclusive em relação a posturas em atividades diárias - tal como pegar objetos do chão - em avaliações feitas após 6 e 12 meses, em comparação a um grupo controle e um placebo, constituído por orientações passadas pelo próprio professor. Esse resultado foi corroborado ainda pela observação durante aulas de educação física, em que observaram maior uso da flexão do joelho, em vez da flexão da coluna, demonstrando a aplicabilidade do programa nas atividades em ambiente natural. Da mesma maneira, Geldhof et al. 14 encontraram resultados positivos quanto ao incremento do conhecimento das posturas em geral, tais como pegar objetos do chão e carregar uma caixa pesada ou um livro, registrando a retenção desse 
conhecimento após um ano. Quanto à postura dormir de lado, não foi encontrado estudo semelhante, uma vez que os programas de educação postural em escolares dão ênfase às posturas utilizadas em sala de aula ou no ambiente escolar.

Em relação ao peso das mochilas, observou-se redução significativa após a sessão educativa, embora, já na primeira avaliação tenha sido verificado que estavam de acordo com a orientação de carregarem até $10 \%$ do peso corporal22. Isso parece dever-se à adoção de um método de ensino em que os alunos têm apenas um caderno para todas as matérias, enquanto os livros permanecem na escola. Outros estudos também encontraram resultados semelhantes $3,28,29$, podendo-se perceber em geral grande adesão às orientações recebidas sobre esse item ${ }^{11}$.

Não foi observada mudança no modelo de mochila empregado; os estudantes que usam o modelo com carrinho alegaram dificuldade no transporte pelo calçamento da rua até a escola, sendo assim optam por transportar na opção duas alças. Ao contrário, Fernandes et al. 3 constataram aumento da utilização do modelo duas alças após a intervenção educativa, e afirmam que a troca para um modelo de mochila não determina a migração para o modo de transporte correspondente, e pode estar relacionada às várias possibilidades de carregar a mochila, independente do modelo.

Quanto à percepção dos pais, a maioria deles responderam que os filhos têm bons hábitos posturais e adotam posturas adequadas nas AVD. Especula-se que esse resultado seja um reflexo do conhecimento que já tinham, mascarando dessa forma os reais hábitos posturais dos filhos. Negrini e Carabalona 30 aplicaram um questionário em escolares da Bélgica para verificar a relação entre o peso da mochila e dores na coluna, bem como o papel dos pais e professores. Concluíram que os pais podem exercitar o cuidado com as mochilas e material escolar desde o início do ano letivo, checando o que seus filhos estão carregando, e certificando-se de que não estão levando material desnecessário para a escola. Professores, estudantes e pais são igualmente envolvidos em determinar o peso da mochila, e todos podem contribuir para reduzi-lo. Cabe destacar a participação dos pais percebida no presente estudo, uma vez que todos responderam ao questionário - ao contrário de um estudo que aponta baixa colaboração ${ }^{3}$.

Os hábitos posturais inadequados são transmitidos de geração a geração, pois estudantes copiam as atitudes dos adultos e, posteriormente, as incorporam ou modificam ${ }^{21}$. Os padrões de postura e movimento, adequados ou não, começam a ser determinados na infância, são praticados na adolescência e logo se tornam habituais na vida adulta. Assim, é essencial fazer as crianças perceberem a importância da postura no cotidiano, conscientizando-as das possíveis conseqüências dos maus hábitos 31 .
O fisioterapeuta que tem a oportunidade de realizar um trabalho de educação postural com escolares deve ter ciência de que isso representa pouco tempo comparado à rotina dos estudantes 32 ; em contrapartida, os professores têm contato diário, facilitando a identificação e correção dos hábitos no âmbito escolar, enquanto os pais têm a tarefa de educá-los nas AVD. Por ter sido realizada apenas uma sessão, a falta de sessões de reforço e de um grupo controle constituíram limitações do presente estudo, de forma que se sugere a inclusão desses itens em estudos futuros. Acredita-se que a realização de reforços por parte dos professores, pais e profissionais da saúde possa desenvolver posturas mais adequadas.

Espera-se também fomentar maior participação de fisioterapeutas no desenvolvimento de programas de promoção da saúde no ambiente escolar, favorecendo aquisição de bons hábitos posturais, promovendo a saúde dos estudantes.

\section{CONCLUSÃO}

Verificou-se que uma sessão educativa não foi suficiente para lograr mudança de hábitos posturais. No entanto, observou-se assimilação do conhecimento de posturas corretas e sua adoção relatada em algumas AVD. Foi constatada boa aceitação da sessão de educação postural por parte dos estudantes.

\section{REFERÊNCIAS}

1 Brasil. Ministério da Saúde. Promoção da saúde: Carta de Ottawa; Declaração de Adelaide, Declaração de Sundsvall e Declaração de Bogotá. Rio de Janeiro: Fiocruz; 1986. p.11-8.

2 Buss PM. Promoção da saúde e qualidade de vida. Cienc Saude Coletiva. 2000;5(1):163-77.

3 Fernandes SMS, Casarotto RA, João SMA. Efeitos de sessões educativas no uso das mochilas escolares em estudantes do ensino fundamental I. Rev Bras Fisioter. 2008;12(6):447-53.

4 Sacco ICN, Mello MCS, Rojas GB, Naki IK, Burgi K, Silveira LTY, et al. Análise biomecânica e cinesiológica de posturas mediante fotografia digital: estudo de casos. Rev Bras Cienc Mov [Brasília]. 2003;11(2):25-33.
5 Pagnussat AS, Paganotto KM. Caracterização da curvatura lombar em escolares na fase do desenvolvimento estrutural. Fisioter Mov. 2008;21(1):39-46.

6 Detsch C. Prevalência de alterações posturais em escolares do ensino médio de São Leopoldo (RS), Brasil [dissertação]. São Leopoldo: Universidade do Vale do Rio dos Sinos; 2005.

7 Detsch C, Luz AMH, Candotti CT, Oliveira DS, Lazaron F, Guimarães LK, et al. Prevalência de alterações posturais em escolares do ensino médio em uma cidade no sul do Brasil. Rev Panam Salud Publica. 2007;21(4):231-8.

8 Bienfait M. Os desequilíbrios estáticos: fisiologia, patologia e tratamento fisioterápico. São Paulo: Summus; 1995. 


\section{Referências (cont.)}

9 Rebolho MCT. Efeitos da educação postural nas mudanças de hábitos em escolares das $1^{\mathrm{a}}$ a $4^{\mathrm{a}}$ séries do ensino fundamental [dissertação]. São Paulo: Faculdade de Medicina, Universidade de São Paulo; 2005.

10 Zapater AR, Silveira DM, Vitta A, Padovani CR, Silva JCP. Postura sentada: a eficácia de um programa de educação para escolares. Cienc Saude Coletiva. 2004;9(1):191-9.

11 Feingold AJ, Jacobs K. The effect of education on backpack wearing and posture in a middle school population. Work. 2002;18(3):287-94.

12 Méndez FJ, Gómez-Conesa A. Postural hygiene program to prevent low back pain. Spine. 2001;26(11):1280-6.

13 Geldhof E, Cardon G, De Bourdeaudhuij I, De Clercq D. Back posture education in elementary schoolchildren:stability of two-year intervention effects. Eura Medicophys. 2007;43(3):369-79.

14 Geldhof E, Cardon G, De Bourdeaudhuij I, De Clercq D. Back posture education in elementary schoolchildren: a 2-year follow-up study. Eur Spine J. 2007;16(6):841-50.

15 Andrade SC, Araujo AGR, Vilar MJP. Escola de Coluna: revisão histórica e sua aplicação na lombalgia crônica. Rev Bras Reumatol. 2005;45(4):224-8.

16 Forsell MZ. The Back School. Spine. 1981;6(1):104-6.

17 Cardon G, De Bourdeaudhuij I, De Clercq D. Back care education in elementary school: a pilot study investigating the complementary role of the class teacher. Patient Educ Couns. 2001;45(3):219-26.

18 Cardon GM, De Clercq DL, De Bourdeaudhuij IM. Back education efficacy in elementary schoolchildren: a 1-year follow-up study. Spine. 2002;27(3):299-305.

19 Cardon G, De Bourdeaudhuij I, De Clercq D. Knowledge and perceptions about back education among elementary school students, teachers, and parents in Belgium. J Sch Health. 2002;72(3):100-6.

20 Rebolho MCT, Cardinalli VA. Posturinha e sua turma: harmonia com a postura. São Paulo: Scortecci; 2007.
21 Santos CIS, Cunha ABN, Braga VP, Saad IAB, Ribeiro MAGO, Conti PBM, et al. Ocorrência de desvios posturais em escolares do ensino público fundamental de Jaguariúna, São Paulo. Rev Paul Pediatr. 2009;(1)74-80.

22 Rodrigues S, Montebelo MIL, Teodori RM. Distribuição da força plantar e oscilação do centro de pressão em relação ao peso e posicionamento do material escolar. Rev Bras Fisioter. 2008;12(1):43-8.

23 Correa AL, Pereira JS, Silva MAG. Avaliação dos desvios posturais em escolares: estudo preliminar. Fisioter Bras. 2005;6(3):175-8.

24 Ainhagne M, Santhiago V. Cadeira e mochila: escolares no processo de desenvolvimento da má postura e possíveis deformidades em crianças de 8-11 anos. Colloquium Vitae [Presidente Prudente]. 2009;1(1):1-7.

25 Limon S, Valinsky LJ, Ben-Shalom Y. Children at risk: risk factors for low-back pain in the elementary school environment. Spine. 2004;29(6):697-702.

26 Ferronato A, Candotti CT, Silveira RP. A incidência de alterações do equilíbrio estático da cintura escapular em crianças entre 7 e 14 anos. Movimento. 1998;5(9):24-30.

27 Skoffer B. Low-back pain in 15- to 16-year-old children in relation to school furniture and carrying of the school bag. Spine. 2007;32(24):E713-7.

28 Ferst NC. O uso da mochila escolar e suas implicações posturais no aluno do colégio militar de Curitiba [dissertação]. Florianópolis: Universidade Federal de Santa Catarina; 2003.

29 Goodgold S, Corcoran M, Gamache D, Gillis J, Guerin J, Coyle JQ. Backpack use in children. Pediatr Phys Ther. 2002;14(3):122-31.

30 Negrini S, Carabalona R. Backpacks on! schoolchildren's perceptions of load, associations with back pain and factors determining the load. Spine. 2002;27(2):187-95.

31 Freire IA, Teixeira TG, Sales CR. Hábitos posturais: diagnóstico a partir de fotografias. Conexões [Campinas]. 2008;6(2):28-41.

32 Gonçalves FD, Catrib AMF, Vieira NFC, Vieira LJES. A promoção da saúde na educação infantil. Interface Comunic Saude Educ. 2008;12(24):181-92. 